\title{
Maternal Morbidity After Previable Prelabor Rupture of Membranes
}

\author{
Sarah K. Dotters-Katz, MD, Alexis Panzer, BA, Matthew R. Grace, MD, Marcela C. Smid, MD, \\ J. Adeolu Keku, BS, Catherine J. Vladutiu, PhD, Kim A. Boggess, MD, and Tracy A. Manuck, MD
}

OBJECTIVE: To identify risk factors for maternal morbidity after previable prelabor rupture of membranes (PROM).

METHODS: We conducted a case-control study of singleton and twin pregnancies complicated by previable PROM (14.0-22.9 weeks of gestation) at a single tertiary care referral institution, 2000-2015. Pregnancies complicated by fetal anomalies, previable PROM within 2 weeks of chorionic villus sampling or amniocentesis, and those with contraindications to expectant management (eg, chorioamnionitis) were excluded. Cases were women with the primary outcome of composite maternal morbidity (defined as having at one or more of the following: sepsis, intensive care unit admission, acute renal insufficiency, uterine curettage, hysterectomy, deep vein thrombosis, pulmonary embolus, blood transfusion, readmission, or maternal death). Controls were women without the primary composite morbidity. Bivariate analysis compared demographic, clinical, and management characteristics of women in the case group and those in the control group. Multivariable logistic regression models were developed to quantify the association between maternal characteristics and composite severe maternal morbidity.

From the Division of Maternal-Fetal Medicine and the University of North Carolina School of Medicine, University of North Carolina, Chapel Hill, North Carolina; and the Division of Maternal-Fetal Medicine, University of Utah, Salt Lake City, Utah.

Supported by 1UL1TR001111 from the Clinical and Translational Science Award program of the National Center for Advancing Translational Sciences, National Institutes of Health.

Presented as a poster at the Society for Maternal-Fetal Medicine Clinical Meeting, February 1-6, 2016, Atlanta, Georgia.

Each author has indicated that he or she has met the journal's requirements for authorship.

Corresponding author: Sarah Dotters-Katz, MD, UNC Maternal-Fetal Medicine, 3010 Old Clinic Building, CB \#7516, Chapel Hill, NC 275997516; email: sarahdk@med.unc.edu.

Financial Disclosure

The authors did not report any potential conflicts of interest.
RESULTS: During the study period, 174 women presented with by previable PROM and were candidates for expectant management. Sixty-five (37\%) women opted for immediate delivery; 109 (63\%) elected expectant management. Twenty-five of 174 (14\%) experienced one or more components of the composite maternal morbidity (cases) and were compared with 149 (86\%) women in the control group. Women in the case group were more not more likely to elect expectant management $(68 \%$ compared with $59 \%, P=.40$ ), but were more likely to be aged 35 years or older (40\% compared with $14 \%, P=.002)$ or to be carrying twins (52\% compared with $16 \%, P<01$ ). In the regression model, twin gestation and age 35 years or older were both significantly associated with increased odds of composite maternal morbidity (odds ratio [OR] 5.62, 95\% confidence interval [CI] 2.21-14.3 and OR 4.00, 95\% CI 1.48-10.8, respectively).

CONCLUSION: Antenatal counseling of women with previable PROM should include that one in seven women experience significant morbidity. Although expectant management was not associated with increased risk in this cohort, women with twins or those aged 35 years or older were at substantially increased risk.

(Obstet Gynecol 2017;129:101-6)

DOI: 10.1097/AOG.0000000000001803

$\mathrm{P}$ relabor rupture of membranes, defined as rupture of membranes before 37 weeks of gestation, complicates $3-4 \%$ of pregnancies. ${ }^{1}$ Previable prelabor rupture of membranes (PROM), defined as rupture of membranes before $230 / 7$ weeks of gestation, is less common and occurs in only 3-4 in 1,000 pregnancies. $^{2,3}$ Options for women with pregnancies complicated by previable PROM include immediate delivery of a previable fetus or expectant management with the goal of achieving fetal viability. Few studies provide guidance for counseling women regarding the maternal risk after previable PROM. Published reports primarily focus on the frequency of infectious morbidities, including chorioamnionitis 
(16.4-71\%), endometritis (4.5-32\%), and maternal sepsis (less than 1 to $9.4 \%$ ). ${ }^{2,4-7}$

There is a paucity of data regarding noninfectious maternal morbidity, including thrombotic events, need for blood transfusion, kidney injury, or need for additional surgical procedures such as uterine curettage or hysterectomy. Additionally, there is little information regarding the maternal risk factors associated with these adverse outcomes. This knowledge limits our ability to counsel women when they present with previable PROM but no clinical indication for delivery. Thus, we sought to describe maternal morbidities (infectious and noninfectious) and identify risk factors for these morbidities after previable PROM.

\section{MATERIALS AND METHODS}

This is a case-control study conducted at a single tertiary care referral center from 2000 to 2015. Women with previable PROM between 14 0/7 weeks and 22 6/7 weeks of gestation were identified from the University of North Carolina-Chapel Hill Women's Hospital delivery log and billing records using the International Classification of Diseases, 9th Revision codes for previable preterm premature rupture of membranes, premature rupture of membranes, and spontaneous abortion $(634,635$, and 637) from 2000 to 2015. All potential participants were reviewed for eligibility by trained researchers and data were independently verified by a second researcher; study data were collected and managed using REDCap electronic data capture tools hosted at the University of North Carolina. ${ }^{8}$

Women were included if rupture of membranes occurred in the absence of labor between $140 / 7$ and 22 6/7 weeks of gestation using the best available gestational dating. Confirmation of membrane rupture required medical record documentation of history, physical examination with vaginal pooling of fluid with Nitrazine or fern testing or both positive, low amniotic fluid volume by ultrasonography, and in some patients, positive results from intraamniotic fluid injection of indigo carmine. Women with labor within 24 hours of rupture, missing delivery data, uncertain date of membrane rupture, those with major fetal congenital anomalies, including renal anomalies associated with oligohydramnios or anhydramnios or membrane rupture within 48 hours of amniocentesis, chorionic villous sampling, or fetal selective reduction were excluded. This study was reviewed and approved by the institutional review board at the University of North Carolina at Chapel Hill.

Women with pregnancies complicated by previable PROM were not offered expectant management if they had evidence of labor, chorioamnionitis, or heavy vaginal bleeding. We classified each woman into one of three categories: 1) those who were candidates for expectant management and elected termination of the pregnancy, 2) those who were candidates for expectant management and elected expectant management, and 3) those who presented with a contraindication for expectant management. Women in the third group were excluded from this analysis.

The primary outcome was defined as a composite of maternal morbidity, defined as at least one of the following: infectious morbidity (maternal sepsis [by using systemic inflammatory response syndrome criteria]) or noninfectious morbidity (maternal intensive care unit admission, acute renal insufficiency [serum creatinine greater than 1.2], need for uterine curettage or hysterectomy, uterine rupture, deep vein thrombosis, pulmonary embolus, need for blood transfusion, need for readmission, or maternal death)..$^{9}$ Other morbidities examined were individual components of the composite as well as postpartum hemorrhage (defined as estimated blood loss greater than 1,000 cc at delivery), endometritis, wound seroma, need for postpartum antibiotics, and having more than one complication. We also assessed a composite of severe maternal morbidity as a secondary outcome, which included hysterectomy, maternal sepsis, pulmonary embolus, intensive care unit admission, acute renal insufficiency (serum creatinine greater than 1.2), blood transfusion of two or more units, or maternal death. Cases were defined as women who were candidates for expectant management who developed any component of the primary composite outcome. Controls were defined as women who candidates for expectant management who did not develop any component of the primary composite outcome.

For women who elected expectant management, the decision to give antibiotics for latency was at the discretion of the admitting health care provider and tocolytics were not used. Demographic, antenatal, and delivery characteristics were compared between women in the case group and those in the control group using $\chi^{2}$, Fisher exact test, Student $t$ test, or Mann-Whitney $U$ test as appropriate. Multivariable logistic regression modeling was used to measure maternal characteristics associated with maternal morbidity (case status). A second model was run to assess risk factors associated with severe maternal morbidity. All variables that were statistically significant in the bivariate analysis at $P<.05$ were included. Analyses were performed using Stata 14.0. 


\section{RESULTS}

Overall, 263 women developed previable PROM from 2000 to 2015 . Of these, 37 (14\%) did not meet inclusion criteria and $52(20 \%)$ had a contraindication for expectant management, leaving 174 women eligible for analysis (Fig. 1). In the study cohort, 25 (14\%, 95\% confidence interval [CI] 9.5-20\%) of 174 experienced the primary outcome and were designated as cases (Table 1). Five women (2.9\%) experienced more than one component of the maternal morbidity composite, and 18 women $(10 \%)$ experienced severe maternal morbidity. Fifty-six women $(39 \%)$ had postpartum hemorrhage and eight $(4.6 \%)$ had endometritis.

Women in the case group were significantly more likely than women in the control group to be 35 years of age or older (40\% compared with 14\%, $P=.002$ ) and to be carrying twins (52\% compared with $16 \%$, $P<.001)$. Other demographic and initial clinical characteristics were similar between groups (Table 2). Multiple gestation was also more common among women with severe maternal morbidity: 9 of 18 women $(50 \%)$ compared with 27 of $156(17 \%)$ women without a morbidity $(P=.003)$. Similarly, 8 of 18 women $(44 \%)$ with severe morbidity but only 23 of $156(15 \%)$ of women without a severe morbidity were aged 35 years or older $(P=.005)$.

Antenatal and delivery characteristics were also similar between women in the case group and those in the control group (Table 3). Women in the case group were not more likely to have elected expectant management $(68 \%$ compared with $59 \%, P=.40)$ nor were women with a severe maternal morbidity more likely to have elected expectant management $(78 \%$ compared with $58 \%, P=.13)$. The median gestational age at delivery for the first neonate (singleton or twin A) and for twin $\mathrm{B}$ also did not differ between the two groups (Table 3). Eleven women attempted an interval twin

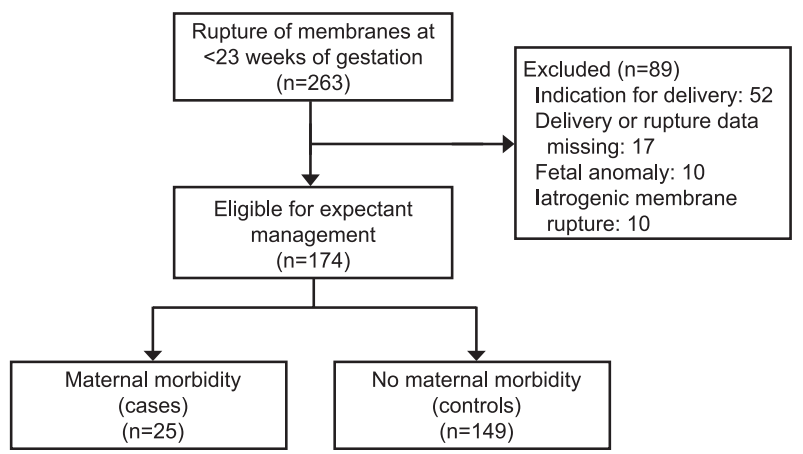

Fig. 1. Study population.

Dotters-Katz. Maternal Morbidity in Membrane Rupture Before 23 Weeks. Obstet Gynecol 2017.
Table 1. Individual Maternal Morbidities Accounting for the 25 Cases of Composite Morbidity*

\section{Complication}

Sepsis

Acute renal injury

Blood transfusion

Intensive care unit admission

Hysterectomy $^{\dagger}$

Deep venous thrombosis

Pulmonary embolus

Death

Readmission

Uterine curettage after delivery $^{\ddagger}$

Other complications

Endometritis

Postpartum hemorrhage

Wound breakdown

More than one complication

Severe maternal morbidity ${ }^{\S}$

\section{No. of Women With Morbidity}

2 (1.2)

$4(2.3)$

$13(7.6)$

$1(0.6)$

$2(1.2)$

0

$1(0.6)$

0

$4(2.3)$

$5(2.9)$

$8(4.6)$

$56(39)$

$2(1.2)$

$5(2.9)$

$18(10)$
Data are $\mathrm{n}(\%)$.

* Composite includes at least one of the following: maternal sepsis, intensive care unit admission, acute renal insufficiency (serum creatinine greater than 1.2), need for uterine curettage or hysterectomy, deep vein thrombosis, pulmonary embolus, need for blood transfusion, need for readmission, or maternal death.

+ Both hysterectomies were for placenta accreta.

* Only includes those women who had uterine curettage after vaginal delivery and cesarean delivery.

$\S$ Severe maternal morbidity includes: hysterectomy, maternal sepsis, pulmonary embolus, intensive care unit admission, acute renal insufficiency (serum creatinine greater than 1.2), blood transfusion of two or more units, or maternal death.

delivery. Of these, 2 of $11(15 \%)$ were in the case group; this rate of maternal morbidity was similar to the overall cohort (25/174 [14\%]). The percentage of women who delivered after 23 weeks of gestation was similar in both groups ( $32 \%$ compared with $29 \%, P=.80$ ).

In the multivariable logistic regression model including twin gestation and maternal age 35 years or older, each was associated with increased odds of maternal morbidity (odds ratio [OR] 5.62, 95\% CI 2.21-14.3 and OR 4.00, 95\% CI 1.48-10.8, respectively). When considering only cases of severe maternal morbidity, these two factors remained significant (Table 4).

\section{DISCUSSION}

We found nearly one in seven women with previable PROM who did not have contraindications to expectant management experienced maternal morbidity. Expectant management was not more common in the 
Table 2. Demographic and Clinical Characteristics Among Women in the Case Group* and Those in the Control Group After Previable Preterm Prelabor Rupture of Membranes, 2000-2015

\section{Characteristic}

Maternal age (y)

Advanced maternal age (35 y or older)

Black race

Private insurance

Nulliparous

Prior preterm birth

Major medical comorbidity ${ }^{\dagger}$

Asthma

Chronic hypertension

Pregestational diabetes

Tobacco use

Recreational drug use

Twin gestation

Any infection in pregnancy ${ }^{\ddagger}$

Bacterial vaginosis

Urinary tract infection

Human papilloma virus
Case Group (Women With

Composite Morbidity) $(n=25)$

$33(25-38)$
$10(40)$
$11(44)$
$14(56)$
$4(16)$
$7(29)$
$7(28)$
$3(12)$
$4(16)$
0
$4(16)$
$2(8.0)$
$13(52)$
$5(20)$
$2(8.0)$
$3(12)$
$2(8.0)$

Control Group (Women Without

Composite Morbidity) $(n=149)$

$\begin{array}{cc}29.5(24-33) & .096 \\ 21(14) & .002 \\ 71(48) & .58 \\ 68(46) & .34 \\ 46(31) & .13 \\ 34(23) & .53 \\ 29(19) & .33 \\ 17(11) & >.99 \\ 13(8.7) & .27 \\ 8(5.7) & .60 \\ 28(19) & >.99 \\ 18(12) & .74 \\ 23(16) & <.001 \\ 60(40) & .053 \\ 14(9.4) & >.99 \\ 18(12) & >.99 \\ 30(20) & .18\end{array}$

Data are median (interquartile range) or $\mathrm{n}(\%)$ unless otherwise specified.

* Women in the case groups defined as at least one of the following: maternal sepsis, intensive care unit admission, acute renal insufficiency (serum creatinine greater than 1.2), need for uterine curettage or hysterectomy, deep vein thrombosis, pulmonary embolus, need for blood transfusion, need for readmission, or maternal death.

${ }^{+}$Includes chronic hypertension, diabetes, and asthma.

₹ Includes bacterial vaginosis, chlamydia, gonorrhea, urinary tract infection, and human papillomavirus.

women in the case group when compared with women in the control group. However, carrying twins compared with a singleton conferred nearly a sixfold increased odds of maternal morbidity and those aged 35 years or older conferred a fourfold increased odds after previable PROM. The most common maternal morbidity in this population was blood transfusion, occurring in $7.6 \%$ of women.

Although data to inform the discussions about neonatal outcomes are increasingly available, there is little information about maternal outcomes. Reddy et al found that as gestational decreased from 33 weeks to 23 weeks, overall risk for severe maternal complications increased. However, only half of that cohort included women with pregnancies complicated by previable PROM and no women with previable PROM before 23 weeks of gestation. ${ }^{10}$ In their study, if delivery occurred between 23 and 27 weeks of gestation, the severe maternal morbidity rate was $11.5 \%$, consistent with our results. Although twin gestation is thought to be associated with an increased risk of pregnancy complications and with prelabor PROM, our study also identifies an increase in maternal risk for women with twins complicated by previable PROM. ${ }^{11-13}$

Strengths of our study include that we examined specific risk factors associated with maternal morbidity and included complications with significant potential to affect maternal health. We clearly defined case status a priori and did not restrict the study to women electing expectant management to better inform counseling for women who present with previable PROM and are faced with a decision about management options (ie, delivery compared with expectant management). Our population is ethnically and economically diverse, increasing the generalizability of our findings.

Despite these strengths, our study has limitations. Like with all retrospective studies, we are limited by data available in the medical record. Because we did not have any patients with gonorrheal or chlamydial infections in our cohort, we cannot comment on the effect of these sexually transmitted infections as risk factors for maternal morbidity. Furthermore, as a tertiary center, our results may have been biased toward more ill women, because those with fewer complications may have delivered at community hospitals. Given our small sample size, we recognize our findings may not be generalizable to the other populations. Also, as a result of the small numbers and retrospective nature, we were also unable to control for factors that may play a role in maternal outcomes, including antibiotics for latency in this population. Current American College of Obstetricians and Gynecologists guidelines suggest 
Table 3. Antenatal and Delivery Characteristics Among Women in the Case Group and Those in the Control Group After Previable Preterm Prelabor Rupture of Membranes 2000-2015

\begin{tabular}{|c|c|c|c|}
\hline Characteristic & $\begin{array}{l}\text { Case Group (Women With } \\
\text { Composite Morbidity)* }(n=25)\end{array}$ & $\begin{array}{l}\text { Control Group (Women Without } \\
\text { Composite Morbidity) }(n=149)\end{array}$ & $P$ \\
\hline Previable preterm PROM GA (wk) & $19.1(17.2-21.6)$ & $20.2(18-21.8)$ & .45 \\
\hline Latency $(\mathrm{wk})^{+\neq}$ & $2.0(0.6-3.1)$ & $2.0(0.6-4.2)$ & .98 \\
\hline Latency for twin B $(w k)^{\neq \S}$ & $2.7(1.4-5.7)$ & $2.0(0.6-4.9)$ & .60 \\
\hline Delivery GA $(w k)^{+}$ & $22.1(19-23.3)$ & $21.3(19.3-23.4)$ & .83 \\
\hline Delivery GA for twin B $(w k)^{\S}$ & $23.5(23-24.2)$ & $23.1(20.4-23.7)$ & .60 \\
\hline Latency antibiotics $\|$ & $8(38)$ & $50(45)$ & .58 \\
\hline Expectant management & $17(68)$ & $88(59)$ & .40 \\
\hline Clinical chorioamnionitis & $5(20)$ & $46(32)$ & .23 \\
\hline WBC at rupture of membranes $(n=42)$ & $13.5(10.9-14.9)$ & $11.5(9.7-14.4)$ & .11 \\
\hline WBC closest to delivery & $17.9(15.9-21.1)$ & $14.5(12.21-18.4)$ & .012 \\
\hline Mode of delivery & & & .94 \\
\hline Vaginal & $17(68)$ & $104(71)$ & \\
\hline Cesarean & $4(16)$ & $20(14)$ & \\
\hline Dilation and evacuation & $4(16)$ & $23(16)$ & \\
\hline $\mathrm{EBL}(\mathrm{CC})$ & $800(500-1,125)$ & $300(150-500)$ & $<.001$ \\
\hline Interval twin delivery & $2(16)$ & $9(39)$ & .26 \\
\hline
\end{tabular}

PROM, preterm prelabor rupture of membranes; GA, gestational age; WBC, white blood cell count; EBL, estimated blood loss.

Data are median (interquartile range) or $\mathrm{n}(\%)$ unless otherwise specified.

* Women in the case group defined as at least one of the following: maternal sepsis, intensive care unit admission, acute renal insufficiency (serum creatinine greater than 1.2), need for uterine curettage or hysterectomy, deep vein thrombosis, pulmonary embolus, need for blood transfusion, need for readmission, or maternal death.

${ }^{+}$For twin gestations, latency is for twin A.

₹ Latency includes only those patients who elected expectant management (17 women in the case group and 88 women in the control group).

$\S$ There were 17 sets of twins in the complications cohort and 19 in the no complications cohort.

" Latency antibiotics are defined as antibiotics given for the specific intention of increasing duration of pregnancy as documented in the medical record.

"considering" this based on level $2 \mathrm{C}$ and $2 \mathrm{~B}$ evidence in women with previable PROM. ${ }^{14}$ Another important limitation of this study is the sample size. Because this was a retrospective cohort, we did not conduct an a priori power calculation. To identify a twofold increase in maternal morbidity associated with expectant management compared with immediate delivery, assuming an $\alpha$ of 0.05 and a $\beta$ of 0.2 , it would require
199 women in each group. Given our study size, we have only $20 \%$ power to detect a $30 \%$ difference in maternal morbidity among women managed expectantly compared with those delivered immediately. Thus, for women with previable PROM, we are unable to make definitive conclusions or recommendations regarding expectant management compared with immediate delivery.

Table 4. Multivariable Logistic Regression Model for Composite Maternal Morbidity and Severe Maternal Morbidity After Previable Prelabor Rupture of Membranes From 2000 to 2015

Adjusted OR

Characteristics associated with maternal morbidity* Maternal age 35 y or older Twin gestation

Characteristics associated with severe maternal morbidity ${ }^{\dagger}$ Maternal age 35 y or older Twin gestation
4.00

4.41

4.36
$95 \% \mathrm{Cl}$

$1.48-10.8$

$2.21-14.2$

$1.51-12.9$

$1.52-12.4$
$P$

.006 $<.01$

.007

.006

$\mathrm{OR}$, odds ratio; $\mathrm{Cl}$, confidence interval.

* Composite includes at least one of the following: maternal sepsis, intensive care unit admission, acute renal insufficiency (serum creatinine greater than 1.2), need for uterine curettage or hysterectomy, deep vein thrombosis, pulmonary embolus, need for blood transfusion, need for readmission, or maternal death.

+ Severe maternal morbidity includes: hysterectomy, maternal sepsis, pulmonary embolus, intensive care unit admission, acute renal insufficiency (serum creatinine greater than 1.2), blood transfusion of two or more units, or maternal death. 
Maternal morbidity after previable PROM is not rare. Approximately $14 \%$ of women who elect for expectant management or immediate delivery after previable PROM experience maternal morbidity. Women with morbidity were not more likely to elect expectant management in this cohort. However, compared with women without morbidity, those with morbidity were more than six times more likely to have twins. The most common maternal complication was blood transfusion. Clinicians may consider incorporating this information when counseling women who present with previable PROM and perhaps considering more aggressive treatment of anemia in those women electing expectant management. Given the rare nature of previable PROM and the limited data on maternal and fetal risks after previable PROM, we propose that the creation of a multicenter prospective registry would substantially improve knowledge about women with previable PROM.

\section{REFERENCES}

1. Goldenberg RL, Culhane JF, Iams JD, Romero R. Epidemiology and causes of preterm birth. Lancet 2008;371:75-84.

2. Waters TP, Mercer BM. The management of preterm premature rupture of the membranes near the limit of fetal viability. Am J Obstet Gynecol 2009;201:230-40.

3. Yeast JD. Preterm premature rupture of the membranes before viability. Clin Perinatol 2001;28:849-60.

4. Hunter TJ, Byrnes MJ, Nathan E, Gill A, Pennell CE. Factors influencing survival in pre-viable preterm premature rupture of membranes. J Matern Fetal Neonatal Med 2012;25:1755-61.
5. Manuck TA, Varner MW. Neonatal and early childhood outcomes following early vs later preterm premature rupture of membranes. Am J Obstet Gynecol 2014;211:308.e1-6.

6. Margato MF, Martins GL, Passini Júnior R, Nomura ML. Previable preterm rupture of membranes: gestational and neonatal outcomes. Arch Gynecol Obstet 2012;285:1529-34.

7. Acaia B, Crovetto F, Ossola MW, Nozza S, Baffero GM, Somigliana E, et al. Predictive factors for neonatal survival in women with periviable preterm rupture of the membranes. J Matern Fetal Neonatal Med 2013;26:1628-34.

8. Harris PA, Taylor R, Thielke R, Payne J, Gonzalez N, Conde JG. Research electronic data capture (REDCap)-a metadatadriven methodology and workflow process for providing translational research informatics support. J Biomed Inform 2009;42:377-81.

9. Levy MM, Fink MP, Marshall JC, Abraham E, Angus D, Cook D, et al. $2001 \mathrm{SCCM} / \mathrm{ESICM} / \mathrm{ACCP} / \mathrm{ATS} / \mathrm{SIS}$ International Sepsis Definitions Conference. Intensive Care Med 2003;29:530-8.

10. Reddy UM, Rice MM, Grobman WA, Bailit JL, Wapner RJ, Varner MW, et al. Serious maternal complications after early preterm delivery (24-33 weeks' gestation). Am J Obstet Gynecol 2015;213:538.e1-9.

11. National Collaborating Centre for Women's and Children's Health (UK). Multiple pregnancy: the management of twin and triplet pregnancies in the antenatal period. NICE Clinical Guidelines, No. 129. London (UK): RCOG Press; 2011.

12. Mercer BM, Crocker LG, Pierce WF, Sibai BM. Clinical characteristics and outcome of twin gestation complicated by preterm premature rupture of the membranes. Am J Obstet Gynecol 1993;168:1467-73.

13. Sela HY, Simpson LL. Preterm premature rupture of membranes complicating twin pregnancy: management considerations. Clin Obstet Gynecol 2011;54:321-9.

14. Periviable birth. Obstetric Care Consensus No. 4. American College of Obstetricians and Gynecologists. Obstet Gynecol 2016;127:e157-69. 